\title{
Association of glycemic status and segmental left ventricular wall thickness in subjects without prior cardiovascular disease: a cross-sectional study
}

Susanne Rospleszcz ${ }^{1 *}$ D, Anina Schafnitzel², Wolfgang Koenig ${ }^{3,4,5}$, Roberto Lorbeer², Sigrid Auweter², Cornelia Huth ${ }^{1,6}$, Wolfgang Rathmann ${ }^{6,7}$, Margit Heier ${ }^{1,8}$, Birgit Linkohr ${ }^{1}$, Christa Meisinger ${ }^{1,6,8}$, Holger Hetterich ${ }^{2}$, Fabian Bamberg ${ }^{2,9}$ and Annette Peters ${ }^{1,5,6}$

\begin{abstract}
Background: Left ventricular (LV) hypertrophy and changes in LV geometry are associated with increased cardiovascular mortality. Subjects with type 2 diabetes have an increased risk of such alterations in cardiac morphology. We sought to assess the association of glycemic status and LV wall thickness measured by cardiac magnetic resonance (CMR), and potential interactions of hypertension and diabetes.

Methods: CMR was performed on 359 participants from a cross-sectional study nested in a population-based cohort (KORA FF4) free of overt cardiovascular disease. Participants were classified according to their glycemic status as either control (normal glucose metabolism), prediabetes or type 2 diabetes. Segmentation of the left ventricle was defined according to the American Heart Association (AHA) 16-segment model. Measurements of wall thickness were obtained at end-diastole and analyzed by linear regression models adjusted for traditional cardiovascular risk factors.

Results: LV wall thickness gradually increased from normoglycemic controls to subjects with prediabetes and subjects with diabetes ( $8.8 \pm 1.4$ vs $9.9 \pm 1.4$ vs $10.5 \pm 1.6 \mathrm{~mm}$, respectively). The association was independent of hypertension and traditional cardiovascular risk factors ( $\beta$-coefficient: $0.44 \mathrm{~mm}$ for prediabetes and $0.70 \mathrm{~mm}$ for diabetes, $p$-values compared to controls: $p=0.007$ and $p=0.004$, respectively). Whereas the association of glycemic status was strongest for the mid-cavity segments, the association of hypertension was strongest for the basal segments.
\end{abstract}

Conclusion: Abnormal glucose metabolism, including pre-diabetes, is associated with increased LV wall thickness independent of hypertension.

Keywords: Cardiac magnetic resonance imaging, Prediabetes, Diabetes, Left ventricular wall thickness, 16-segment model

\footnotetext{
* Correspondence: susanne.rospleszcz@helmholtz-muenchen.de

${ }^{1}$ Institute of Epidemiology, Helmholtz Zentrum München, German Research

Center for Environmental Health, Ingolstaedter Landstrasse 1, 85764

Neuherberg, Germany

Full list of author information is available at the end of the article
}

(c) The Author(s). 2018 Open Access This article is distributed under the terms of the Creative Commons Attribution 4.0 International License (http://creativecommons.org/licenses/by/4.0/), which permits unrestricted use, distribution, and reproduction in any medium, provided you give appropriate credit to the original author(s) and the source, provide a link to the Creative Commons license, and indicate if changes were made. The Creative Commons Public Domain Dedication waiver (http://creativecommons.org/publicdomain/zero/1.0/) applies to the data made available in this article, unless otherwise stated. 


\section{Background}

Abnormal cardiac morphology, such as left ventricular (LV) hypertrophy and altered geometry, represents a risk factor for increased cardiovascular mortality and morbidity $[1,2]$.

Increased LV wall thickness is considered as an adaptive response to augmented wall stress caused by pressure overload. Short-term increase in wall thickness can therefore be regarded as a beneficial adaptation in order to maintain oxygen demand and contractile function of the heart [3]. However, a persistent increase in wall thickness leads to impaired myocardial relaxation and subsequently to decreased diastolic function, [4] which is associated with diastolic heart failure and other adverse cardiovascular outcomes [5]. The exact pathophysiological pathways of the transition from compensatory response to a detrimental chronic condition are not yet fully understood.

Chronic hypertension and the resulting increased hemodynamic load are a major risk factor for cardiac remodeling. However, metabolic factors, including diabetes status, play an important role [6-9]. Multiple studies have used echocardiography to analyze the potential impact of glycemic status on measures of LV mass and geometric patterns. Although most studies found higher values of LV mass in people with abnormal glucose metabolism, these associations were often attenuated by the presence of other traditional cardiovascular risk factors, especially elevated blood pressure [10-15]. Moreover, the prognostic utility of LV mass for CVD events in people with diabetes also depends on other metabolic factors [16-18].

These findings raise the question whether these measurements of LV mass and geometric patterns are detailed enough to describe the complex interplay between glycemic status and blood pressure on cardiac structure. Cardiac magnetic resonance imaging (CMR) has now become the gold standard for the measurement of myocardial mass and volumes [19,20] and delivers a more detailed characterization of cardiac morphology than echocardiography, thereby allowing more precise insights into the mechanisms of LV remodeling.

In our initial analyses, using CMR to derive measures of LV geometry and function, we had observed an increased myocardial mass in subjects with abnormal glucose metabolism, but the difference disappeared after adjustment for major cardiovascular risk factors [21]. We therefore aim to elucidate the impact of glycemic status on regional LV remodeling and further analyze its potential interaction with blood pressure.

\section{Methods}

\section{Study sample}

The study sample is a subsample of the second follow-up of the population-based KORA ("Cooperative
Health Research in the Region of Augsburg") S4 cohort (KORA FF4). The major focus of the substudy is the analysis of subclinical cardiovascular disease by whole-body magnetic resonance imaging (MRI).

Recruitment and eligibility criteria for the KORA studies have been described elsewhere [22]. The KORA FF4 study was conducted between 2013 and 2014 and included 2279 of the originally recruited 4261 KORA S4 participants. Of these, 400 subjects participated in the MRI substudy who were eligible and willing to undergo whole-body MRI. The detailed participant flow and exclusion criteria have been described previously [21].

Additionally, we excluded 31 subjects with incomplete measurements of any AHA segment due to low image quality and subsequently excluded 10 subjects with visible Late Gadolinium Enhancement.

\section{Covariate assessment}

Glycemic status was defined as known diabetes, either self-reported or defined by current use of glucose-lowering medication, and in participants without known diabetes, it was determined by a standard 2-h oral glucose tolerance test (OGTT). According to the 1999 WHO criteria [23], subjects with fasting serum glucose levels $\geq 7.0 \mathrm{mmol} / \mathrm{l}$ or OGTT 2 -h serum glucose levels $\geq 11.1 \mathrm{mmol} / \mathrm{l}$ were also classified as having diabetes. Isolated impaired fasting glucose (iIFG) was defined as fasting glucose $\geq 6.1$ but $<6.9 \mathrm{mmol} / \mathrm{l}$ and 2-h glucose $<7.8 \mathrm{mmol} / \mathrm{l}$. Isolated impaired glucose tolerance (iIGT) was defined as fasting glucose < $6.1 \mathrm{mmol} / \mathrm{l}$ and 2 -h glucose $\geq 7.8$ but $<11.1 \mathrm{mmol} / \mathrm{l}$. Subjects with iIFG, iIGT or with both conditions were classified as having prediabetes. Subjects with fasting glucose $<6.1 \mathrm{mmol} / \mathrm{l}$ and 2-h glucose $<7.8 \mathrm{mmol} / \mathrm{l}$ were considered controls.

Hypertension was defined as current antihypertensive treatment and/or systolic/diastolic blood pressure above 140/90 mmHg. Prehypertension was defined as systolic/ diastolic blood pressure above 120/80 mmHg. Subjects were classified as smokers if they reported current regular or sporadic cigarette smoking. Cholesterol, serum glucose, serum insulin and Hba1c were determined by standard methods as described in Additional file 1: Text S1.

\section{CMR outcome assessment}

Magnetic resonance imaging was performed at a 3 Tesla Magnetom Skyra (Siemens AG, Healthcare Sector, Erlangen Germany) using a 18 channel body coil in combination with the table-mounted spine matrix coil. The whole-body MRI protocol comprised several sequences as described previously [21].

Imaging of cardiac function and morphology was performed using cine steady-state free precession (cine-SSFP) sequences in the short axis with a stack of 
10 layer and 25 phases per cardiac cycle as well as in a 4-chamber view (echo time $1.46 \mathrm{~ms}$, repetition time $29.97 \mathrm{~ms}$, in-plane voxel size $1.5 \times 1.5 \mathrm{~mm}$, flip angle $62-63^{\circ}$, field-of-view $297 \times 360 \mathrm{~mm}$, matrix size $240 \times$ $160 \mathrm{~mm}$, slice thickness $8 \mathrm{~mm}$ ).

The cine-SSFP sequences were then analyzed semi-automatically using cvi42 software (Circle Cardiovascular Imaging, Calgary, Canada) by two readers unaware of the subject's glycemic status and all other clinical covariates. In the 4-chamber view, apex and base of the LV were first manually selected, followed by automatic border detection of the LV endocardial and epicardial border in the short axis. Borders were corrected manually, if necessary. The basal slice was selected when at least $50 \%$ of the LV cavity was surrounded by myocardium at end-diastole. Papillary muscles and trabeculations were excluded from the myocardial area and included in the blood pool. To assess intraobserver agreement, measurements of 25 randomly chosen subjects were repeated by the first reader. Interobserver agreement was assessed on 52 subjects who were measured by the first and the second reader. Intra- and Interobserver agreement were calculated by the Intraclass Correlation Coefficient (ICC).

Mean wall thickness was measured at the end of diastole for each segment according to the American Heart Association (AHA) 16-segment model [24]. Measurements of the single segments are visualized in a polar plot according to glycemic status. For further statistical analysis, segments are grouped according to level (basal: AHA segments 1-6; mid-cavity: AHA segments 7-12; apical: AHA segments 12-16) and region (lateral: AHA segments 5, 6, 11, 12 and 16; septal: AHA segments 2, 3, 8 and 9; anterior: AHA segments 1, 7 and 13; inferior: AHA segments 4, 10 and 15) [24].

\section{Statistical analysis}

Main demographic and cardiovascular characteristics of the participants are reported as arithmetic means with standard deviations for continuous variables and counts and percentages for categorical variables. Differences in wall thickness between the glycemic groups were assessed by one-way ANOVA. A linear regression model including glycemic status, age, sex, Body Mass Index (BMI), systolic blood pressure, total cholesterol, use of antihypertensive medication and smoking status was calculated to determine the association of glycemic status to the respective wall thickness variable. The same model without adjustment for systolic blood pressure was calculated for hypertension. Additionally, linear regression models with multiplicative terms between glycemic status and systolic blood pressure were computed to discover any interaction effects.
As the MRI sample is a non-representative subsample of a population based cohort, we used appropriate sampling weights to render the sample more representative of the full eligible underlying cohort. Weighting was based on glycemic status, age and sex. Details of the weighting procedure are presented in Additional file 1: Text S2.

$P$-values $<0.05$ were considered to denote statistical significance. All analyses were done with $\mathrm{R}$ version 3.2.1 (R Core Team, Vienna, Austria).

The KORA FF4 study was approved by the ethics committee of the Bavarian Chamber of Physicians, Munich; the MRI substudy by the institutional review board of the Ludwig-Maximilians-University Munich. The investigations were carried out in accordance with the Declaration of Helsinki, including written informed consent of all participants.

\section{Results \\ Study population}

The sample of 359 subjects comprised 223 normoglycemic controls (62\%), 92 subjects with prediabetes (26\%) and 44 subjects with diabetes (12\%) as presented in Table 1. Of those, 15 diabetes cases were diagnosed based on the results of OGTT. In subjects with established diabetes the median duration of diabetes was 7.0 years.

\section{Intra- and Interobserver agreement}

The ICCs for intraobserver and interobserver agreement were 0.93 and 0.94 for mean wall thickness, respectively. ICCs for single segments are presented in Additional file 1: Figure S1.

\section{Comparison of wall thickness according to glycemic status} Mean wall thickness of all AHA segments in the whole sample was $9.1 \mathrm{~mm}$ ( \pm standard deviation: $1.5 \mathrm{~mm}$ ). The polar plots in Fig. 1 display the wall thickness of the individual AHA segments for the three glycemic groups.

We found a gradual increase in wall thickness from controls through prediabetes to diabetes for all classes of segments grouped by level and region. The differences between the glycemic groups were statistically significant in univariate analysis for all analyzed segment classes (Table 2).

\section{Association of glycemic status and wall thickness independent of confounding factors}

After adjustment for additional covariates as detailed above, prediabetes and diabetes were independently associated to increased wall thickness (prediabetes: $\beta: 0.44 \mathrm{~mm}$, 95\%-CI: $[0.12 \mathrm{~mm}, 0.75 \mathrm{~mm}$ ], diabetes: $\beta: 0.70 \mathrm{~mm}$, 95\%-CI: [0.23 mm, $1.17 \mathrm{~mm}]$ ). Associations held true for most segment classes according to level and region as 
Table 1 Demographic characteristics of study participants

\begin{tabular}{|c|c|c|c|c|}
\hline & All & Control & Prediabetes & Type 2 diabetes \\
\hline & $N=359$ & $N=223$ & $N=92$ & $N=44$ \\
\hline Age (years) & $56.1 \pm 9.1$ & $54.3 \pm 8.9$ & $58.1 \pm 8.8$ & $61.4 \pm 8.3$ \\
\hline Male gender & $205(57.1 \%)$ & $115(51.6 \%)$ & $58(63.0 \%)$ & $32(72.7 \%)$ \\
\hline $\mathrm{BMI}\left(\mathrm{kg} / \mathrm{m}^{2}\right)$ & $27.9 \pm 4.8$ & $26.5 \pm 4.2$ & $30.3 \pm 4.6$ & $30.2 \pm 5.1$ \\
\hline Systolic blood pressure (mmHg) & $120.3 \pm 16.9$ & $116.5 \pm 15.2$ & $124.7 \pm 15.5$ & $130.1 \pm 21.2$ \\
\hline Diastolic blood pressure $(\mathrm{mmHg})$ & $75.3 \pm 10.1$ & $73.7 \pm 9.2$ & $78.0 \pm 9.7$ & $78.0 \pm 13.2$ \\
\hline Prehypertension & $94(26.2 \%)$ & $61(27.4 \%)$ & $26(28.3 \%)$ & $7(15.9 \%)$ \\
\hline Hypertension & $117(32.6 \%)$ & $45(20.2 \%)$ & $41(44.6 \%)$ & $31(70.5 \%)$ \\
\hline Fasting glucose (mmol/L) & $5.7 \pm 1.3$ & $5.2 \pm 0.4$ & $5.9 \pm 0.6$ & $8.0 \pm 2.3^{\mathrm{a}}$ \\
\hline Fasting insulin (pmol/L) & $65.4 \pm 41.1$ & $51.6 \pm 26.7$ & $86.9 \pm 44.6$ & $91.7 \pm 60.0^{b}$ \\
\hline $\mathrm{HbA1c}(\%)$ & $5.6 \pm 0.7$ & $5.3 \pm 0.3$ & $5.6 \pm 0.3$ & $6.7 \pm 1.4$ \\
\hline Total cholesterol (mmol/L) & $5.6 \pm 1.0$ & $5.6 \pm 0.9$ & $5.8 \pm 0.8$ & $5.5 \pm 1.2$ \\
\hline $\mathrm{HDL}$ cholesterol $(\mathrm{mmol} / \mathrm{L})$ & $1.6 \pm 0.5$ & $1.7 \pm 0.5$ & $1.5 \pm 0.4$ & $1.4 \pm 0.4$ \\
\hline LDL cholesterol (mmol/L) & $3.6 \pm 0.9$ & $3.6 \pm 0.8$ & $3.7 \pm 0.8$ & $3.5 \pm 1.1$ \\
\hline Triglycerides (mmol/L) & $1.5 \pm 1.0$ & $1.2 \pm 0.7$ & $1.7 \pm 1.0$ & $2.3 \pm 1.4$ \\
\hline \multicolumn{5}{|l|}{ Smoking } \\
\hline Never-smoker & $130(36.2 \%)$ & $88(39.5 \%)$ & $28(30.4 \%)$ & $14(31.8 \%)$ \\
\hline Ex-smoker & $156(43.5 \%)$ & $87(39.0 \%)$ & $46(50.0 \%)$ & $23(52.3 \%)$ \\
\hline Smoker & $73(20.3 \%)$ & $48(21.5 \%)$ & $18(19.6 \%)$ & $7(15.9 \%)$ \\
\hline Antihypertensive medication & $85(23.7 \%)$ & $35(15.7 \%)$ & $28(30.4 \%)$ & $22(50.0 \%)$ \\
\hline
\end{tabular}

Values are arithmetic means \pm standard deviations for continuous variables and number of subjects (percentage of respective group) for categorical outcomes a Based on $N=43$ subjects with type 2 diabetes

${ }^{\text {b } B a s e d}$ on $N=42$ subjects with type 2 diabetes

presented in Table 3. The strongest associations were found for the mid-cavity segments and the anterior segments.

\section{Effects of weighting}

Additional file 1: Table S1 shows the characteristics of the underlying eligible cohort that was used for the calculation of sampling weights.

Comparing the MRI sample to the whole cohort, there was an overrepresentation of subjects with prediabetes
(26\% in the sample vs $12 \%$ in the cohort) and subjects with diabetes ( $12 \%$ in the sample vs $10 \%$ in the cohort). Additionally, the proportion of males was higher in the MRI sample compared to the underlying cohort, whereas mean age was similar.

Results of the unweighted analysis are presented in Additional file 1: Table S2. Associations that were statistically significant in the weighted analysis were also significant in the unweighted analysis. The size of the estimates was comparable; however as sampling weights

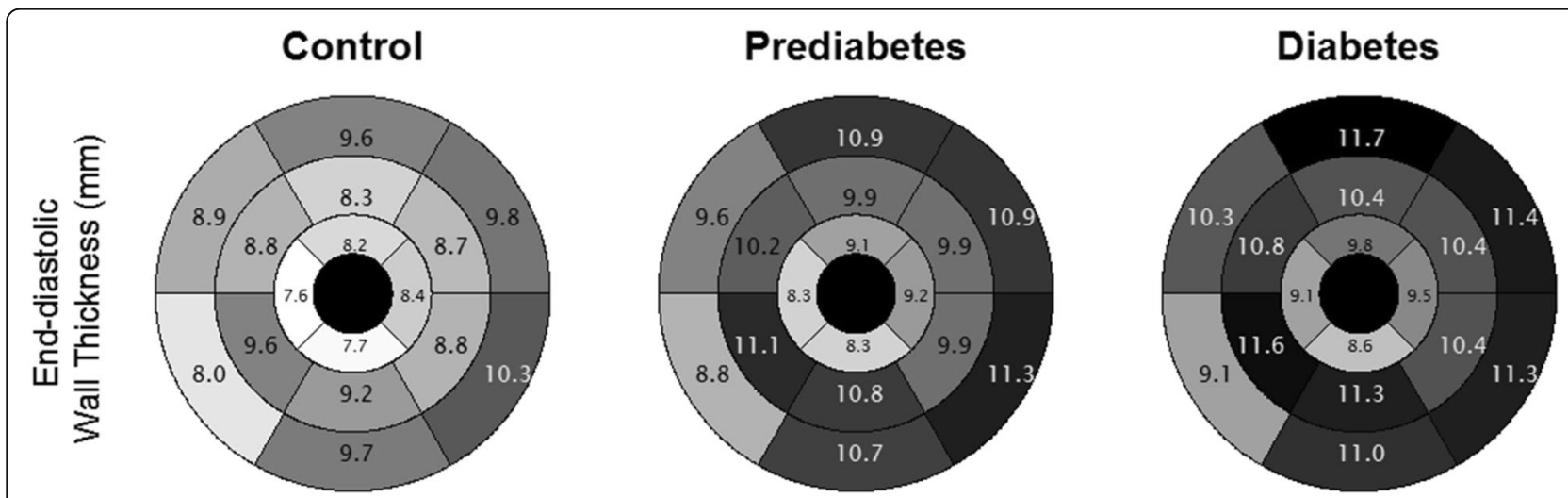

Fig. 1 Left ventricular wall thickness (mm) of 16 AHA segments for control, prediabetes and diabetes group 
Table 2 Mean wall thickness grouped by level and region and myocardial mass

\begin{tabular}{|c|c|c|c|c|c|c|}
\hline \multirow{2}{*}{\multicolumn{2}{|c|}{$\begin{array}{ll}\text { All } \\
& N=359 \\
\end{array}$}} & \multirow{2}{*}{$\begin{array}{l}\text { Control } \\
N=223\end{array}$} & \multirow{2}{*}{$\begin{array}{l}\text { Prediabetes } \\
N=92\end{array}$} & \multirow[t]{2}{*}{$P$-value } & Type 2 diabetes & \multirow[t]{2}{*}{$P$-value } \\
\hline & & & & & $N=44$ & \\
\hline \multicolumn{7}{|c|}{ Wall thickness (mm): arithmetic mean of } \\
\hline All segments & $9.1 \pm 1.5$ & $8.8 \pm 1.4$ & $9.9 \pm 1.4$ & $<0.001$ & $10.5 \pm 1.6$ & $<0.001$ \\
\hline Basal segments & $9.6 \pm 1.6$ & $9.4 \pm 1.6$ & $10.4 \pm 1.6$ & $<0.001$ & $10.8 \pm 1.7$ & $<0.001$ \\
\hline Mid segments & $9.2 \pm 1.8$ & $8.9 \pm 1.6$ & $10.3 \pm 1.8$ & $<0.001$ & $10.9 \pm 2.1$ & $<0.001$ \\
\hline Apical segments & $8.2 \pm 1.5$ & $8.0 \pm 1.4$ & $8.7 \pm 1.5$ & $<0.001$ & $9.3 \pm 1.6$ & $<0.001$ \\
\hline Lateral segments & $9.8 \pm 1.6$ & $9.3 \pm 1.5$ & $10.3 \pm 1.4$ & $<0.001$ & $10.8 \pm 1.8$ & $<0.001$ \\
\hline Septal segments & $9.1 \pm 1.6$ & $8.7 \pm 1.5$ & $9.7 \pm 1.4$ & $<0.001$ & $10.3 \pm 1.6$ & $<0.001$ \\
\hline Anterior segments & $9.4 \pm 1.9$ & $8.8 \pm 1.7$ & $10.0 \pm 1.7$ & $<0.001$ & $10.8 \pm 2.2$ & $<0.001$ \\
\hline Inferior segments & $9.4 \pm 1.5$ & $9.0 \pm 1.5$ & $9.9 \pm 1.4$ & $<0.001$ & $10.4 \pm 1.4$ & $<0.001$ \\
\hline Myocardial mass $\left(\mathrm{g} / \mathrm{m}^{2}\right)$ & $70.0 \pm 13.9$ & $69.0 \pm 13.6$ & $72.3 \pm 13.0$ & 0.1 & $75.9 \pm 16.0$ & 0.019 \\
\hline
\end{tabular}

$P$-values are obtained from one-way ANOVA and Bonferroni corrected for the repeated comparisons to the control group

introduce additional variation in the data, confidence intervals for the weighted analysis were wider than for the unweighted analysis. Model diagnostics such as residual distribution were similar between the weighted and unweighted analysis. Taken together, the evidence suggests that the analytical model was correctly specified [25] and although the weighted estimates are conceptually more precise, as they relate to the underlying cohort, the actual differences between weighted and unweighted estimates were small.

\section{Association of specific prediabetes subgroups and wall thickness}

We further differentiated prediabetes status into subjects with ifFG $(N=35,9.7 \%$ of total sample and $38.1 \%$ of subjects with prediabetes), iIGT ( $N=41,11.4 \%$ of total sample and $44.6 \%$ of subjects with prediabetes) and both IFG + IGT $(N=16,4.5 \%$ of total sample and $17.4 \%$ of subjects with prediabetes). Though there were differences in mean wall thickness according to segment classes between the prediabetes subgroups there was no gradual increase from iIFG to iIGT and IFG + IGT (See Additional file 1: Figure S2 and Table S3).

\section{Association of hypertension and wall thickness independent of confounding factors}

Prehypertension and hypertension were significantly associated with increased wall thickness (prehypertension: $\beta$ : $0.48 \mathrm{~mm}, 95 \%$-CI: [0.17 mm, $0.79 \mathrm{~mm}$ ], hypertension: $\beta$ : $0.67 \mathrm{~mm}, 95 \%$-CI: [0.31 mm, $1.02 \mathrm{~mm}$ ) after adjustment for additional covariates. The strongest associations were seen for the basal segments and the septal segments as presented in Table 4. Notably, there was also a significant association of hypertension to myocardial mass.

\section{Interaction of glycemic status and blood pressure}

As displayed in Fig. 2 we found no interaction of glycemic status and systolic blood pressure for mean wall thickness averaged over all segments. Marginal effects of glycemic status, i.e. associations of prediabetes and

Table 3 Association of glycemic status and wall thickness

\begin{tabular}{|c|c|c|c|c|c|c|}
\hline & \multicolumn{3}{|c|}{ Prediabetes } & \multicolumn{3}{|l|}{ Diabetes } \\
\hline & Estimate & $95 \%-\mathrm{Cl}$ & $P$-value & Estimate & $95 \%-\mathrm{Cl}$ & $P$-value \\
\hline \multicolumn{7}{|c|}{ Wall thickness (mm): arithmetic mean of } \\
\hline All segments & 0.44 & {$[0.12,0.75]$} & 0.007 & 0.70 & {$[0.23,1.17]$} & 0.004 \\
\hline Basal segments & 0.33 & {$[-0.05,0.70]$} & 0.087 & 0.51 & {$[0.02,0.99]$} & 0.040 \\
\hline Mid segments & 0.61 & {$[0.20,1.02]$} & 0.004 & 0.86 & {$[0.28,1.45]$} & 0.004 \\
\hline Apical segments & 0.34 & {$[-0.04,0.73]$} & 0.080 & 0.74 & {$[0.23,1.24]$} & 0.005 \\
\hline Lateral segments & 0.46 & {$[0.09,0.83]$} & 0.014 & 0.65 & {$[0.14,1.16]$} & 0.013 \\
\hline Septal segments & 0.35 & {$[0.05,0.65]$} & 0.023 & 0.64 & {$[0.18,1.10]$} & 0.006 \\
\hline Anterior segments & 0.52 & {$[0.10,0.93]$} & 0.015 & 0.98 & {$[0.38,1.59]$} & 0.002 \\
\hline Inferior segments & 0.45 & {$[0.11,0.79]$} & 0.009 & 0.58 & {$[0.11,1.04]$} & 0.016 \\
\hline Myocardial mass $\left(\mathrm{g} / \mathrm{m}^{2}\right)$ & -0.11 & {$[-3.51,3.28]$} & 0.948 & 0.56 & {$[-4.94,6.07]$} & 0.841 \\
\hline
\end{tabular}

Estimates from linear regression models adjusted for age, sex, BMl, systolic blood pressure, total cholesterol, use of antihypertensive medication and smoking status 
Table 4 Association of hypertension and wall thickness

\begin{tabular}{|c|c|c|c|c|c|c|}
\hline & \multicolumn{3}{|c|}{ Prehypertension } & \multicolumn{3}{|c|}{ Hypertension } \\
\hline & Estimate & $95 \%-\mathrm{Cl}$ & $P$-value & Estimate & $95 \%-\mathrm{Cl}$ & $P$-value \\
\hline \multicolumn{7}{|c|}{ Wall thickness (mm): arithmetic mean of } \\
\hline All segments & 0.48 & {$[0.17,0.79]$} & 0.003 & 0.67 & {$[0.31,1.02]$} & $<0.001$ \\
\hline Basal segments & 0.63 & {$[0.28,0.98]$} & $<0.001$ & 0.83 & {$[0.42,1.24]$} & $<0.001$ \\
\hline Mid segments & 0.35 & {$[-0.01,0.71]$} & 0.057 & 0.70 & {$[0.26,1.14]$} & 0.002 \\
\hline Apical segments & 0.44 & {$[0.02,0.86]$} & 0.043 & 0.38 & {$[-0.01,0.77]$} & 0.060 \\
\hline Lateral segments & 0.37 & {$[0.03,0.71]$} & 0.036 & 0.52 & {$[0.12,0.92]$} & 0.012 \\
\hline Septal segments & 0.59 & {$[0.29,0.89]$} & $<0.001$ & 0.84 & {$[0.48,1.19]$} & $<0.001$ \\
\hline Anterior segments & 0.44 & {$[0.02,0.86]$} & 0.039 & 0.77 & {$[0.34,1.19]$} & $<0.001$ \\
\hline Inferior segments & 0.51 & {$[0.14,0.88]$} & 0.007 & 0.54 & {$[0.16,0.92]$} & 0.006 \\
\hline Myocardial mass $\left(\mathrm{g} / \mathrm{m}^{2}\right)$ & 3.18 & {$[-0.02,6.39]$} & 0.053 & 6.16 & {$[2.19,10.12]$} & 0.003 \\
\hline
\end{tabular}

Estimates from linear regression models adjusted for age, sex, BMI, glycemic status, total cholesterol, use of antihypertensive medication and smoking status
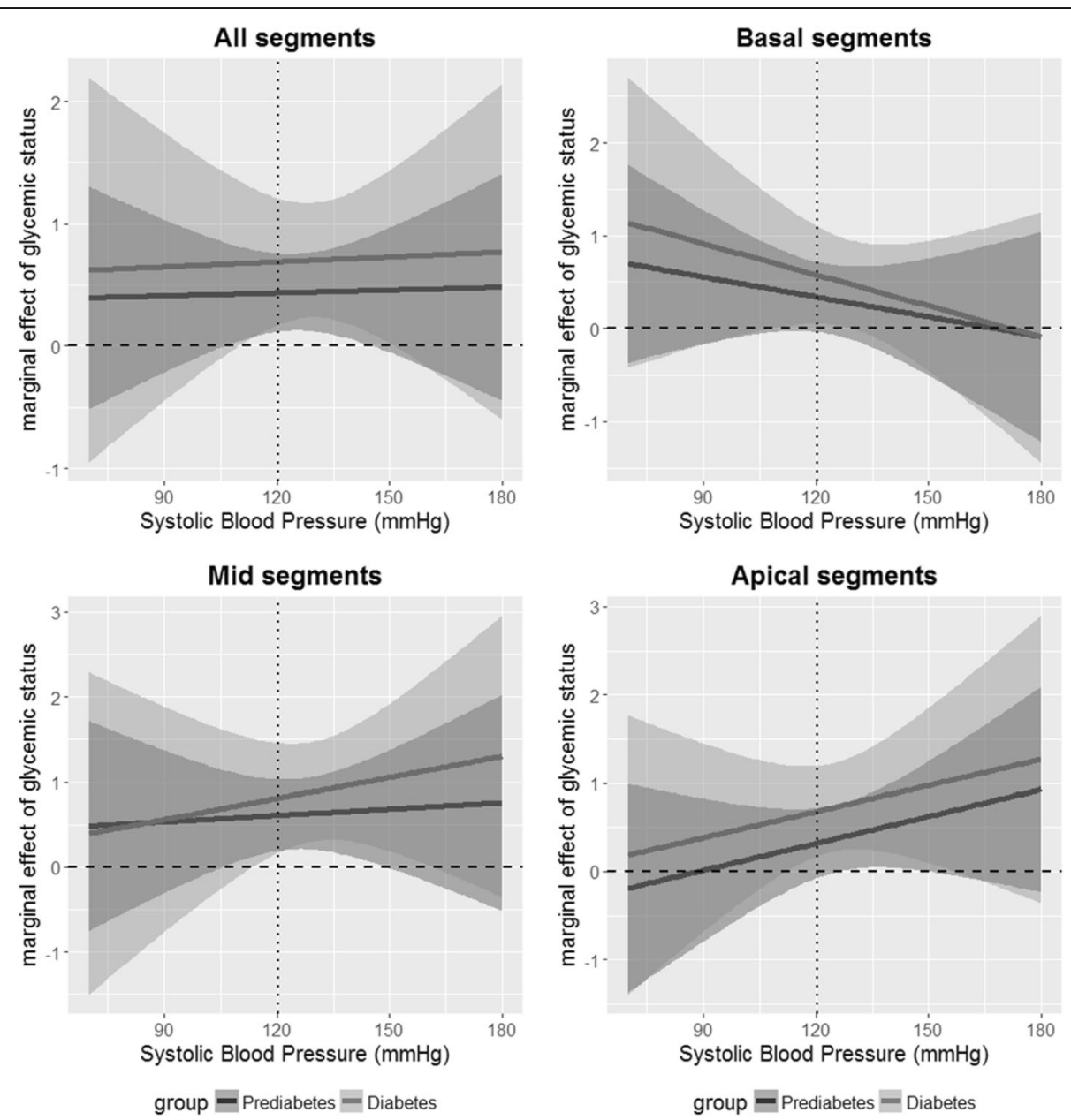

Fig. 2 Marginal effects of glycemic status on wall thickness for multiplicative interactions with systolic blood pressure. Marginal effects indicate the size of the association of the respective group (prediabetes or diabetes) with wall thickness for a specific value of systolic blood pressure. Displayed are the marginal effects of prediabetes (solid line, dark grey) and diabetes (solid line, light grey) and the respective $95 \%$ confidence interval for a grid of possible values of systolic blood pressure (range in data: 73-179.5 $\mathrm{mmHg}$ ). The arithmetic mean is indicated by a dotted line. The dashed line indicates the line of no effect 
diabetes with wall thickness for a specific value of systolic blood pressure, remained constant over the range of possible blood pressure values. However, for basal segments, there was a decreasing marginal effect of glycemic status with rising blood pressure, whereas for mid-cavity and apical segments the marginal effect of glycemic status was increasing with rising blood pressure.

\section{Discussion}

Increased LV wall thickness is associated with a higher risk for cardiovascular outcomes. Recent findings from the Framingham study showed that a 0.1 unit increase in relative wall thickness was accompanied by a $59 \%$ increase in the hazard for cardiovascular disease [1]. A detailed assessment of LV geometry based on regional wall thickness can predict risk of incident cardiovascular disease [26]. Given these implications, it is of major importance to determine modifiable risk factors for increased wall thickness.

Our findings from this cross-sectional study show that (i) type 2 diabetes is associated to increased LV wall thickness, independent of traditional cardiovascular risk factors and especially independent of hypertension, (ii) the independent association of abnormal glucose homeostasis to cardiac structure is already present in prediabetes, (iii) individual LV segments are differently affected by hypertension and glycemic status. Thereby, we could demonstrate that the more specific evaluation of CMR derived regional LV wall thickness unveils associations that cannot be detected when assessing LV mass alone.

Our results therefore support and extend findings from other established population-based studies. In the Atherosclerosis Risk in the Community (ARIC) study, Skali et al. found that mean and relative LV wall thickness were elevated in subjects with diabetes independent of systolic blood pressure. To a certain extent, wall thickness was already increased in subjects with prediabetes [27]. In the Framingham Offspring Cohort, Velageti et al. [28] found an increasing CMR derived relative wall thickness across glycemia categories. After multivariable adjustment, the association remained significant in men. In the Multiethnic Study of Atherosclerosis (MESA), an association between CMR-derived LV mass and diabetes was found that was independent of blood pressure; however no measurements of segmental wall thickness were taken [29]. On the other hand, Bertoni et al. measured mid-cavity segments and found an increasing wall thickness for Caucasian subjects with normal glucose metabolism to subjects with iIFG and type 2 diabetes. The differences were not significant after adjustment for other cardiovascular risk factors [30]. Another study found an association of glycemic status to LV mass, but not to (relative or mean) wall thickness [31]. In our sample, we showed that the increased LV mass in subjects with diabetes was attributable to hypertension but there are independent regional associations of diabetes and blood pressure in segmental wall thickness.

Regarding the prediabetic state, recent evidence from the CARDIA study implies that longer exposure to abnormal glucose tolerance, longer duration of diabetes, and early onset of diabetes leads to more unfavorable remodeling [32]. Analyses from the Strong Heart Study demonstrated increased LV mass in Native Americans with impaired glucose tolerance, however the finding was less definitive for measures of wall thickness [33]. Our findings corroborate that prediabetes, defined as either iIGT, iIFG or IFG + IGT, is independently associated to increased wall thickness. Our sample size was probably too small to detect gradual effects of these different prediabetic groups.

Disentangling the associations of blood pressure and glycemic status proves to be complicated. In the Strong Heart Study, LV mass of subjects with diabetes but without hypertension was significantly lower compared to those subjects with both conditions, whereas relative wall thickness was not different [34]. In the HyperGEN study comprising only hypertensive subjects, diabetes was independently associated to increased LV mass [35]. A recent Chinese study suggested additive effects of diabetes and hypertension to LV remodeling [36]. Although blood pressure reduction appears to be an effective way of lowering the risk of cardiovascular disease in hypertensive patients with increased LV mass, [37] these treatments do not seem to be as effective in patients with diabetes [38]. In our study, we found a decreasing marginal effect of both prediabetes and diabetes with rising blood pressure for the basal segments, whereas the marginal effect for apical and mid segments was increasing. Thus we could further characterize the complex interplay of blood pressure and glycemic status and its impact on cardiac geometry.

The exact mechanisms of impaired glucose metabolism on LV geometry remain to be identified. Increased LV stiffness, induced by an accumulation of collagen and advanced glycation end products and subsequent fibrosis in diabetic cardiomyopathy have been suggested to contribute to LV remodeling [39, 40]. Also, a decreased myocardial perfusion reserve in subjects with diabetes induced by an impaired myocardial blood flow has been shown to be correlated with LV torsion and strain [41]. Recent findings imply that myocardial steatosis, excess storage of cardiac triglycerides, and impaired myocardial energetics are associated with concentric LV remodeling [42]. However, our study is limited in this respect as it cannot explain the pathophysiological mechanisms behind the association of glycemic status on regional LV geometry. 
The values of LV wall thickness reported here, as measured by short-axis cine SSFP, substantially exceed the reference values according to the 16-segment model suggested by other groups [43, 44]. These reference values have been obtained from healthy subjects with a low-risk profile for developing cardiovascular disease, excluding smokers, and people with hypertension or diabetes. Given the major impact of these risk factors on wall thickness, it is plausible that our study found larger values for the control group.

For this nested cross-sectional study, we used a well-characterized population-based cohort. Highly standardized measurements and validation allowed us to precisely define covariates and glycemic status. Furthermore, using adequate sampling weights we were able to relate our results to the full underlying cohort.

Limitations of our study include its cross-sectional design that precludes causal inference. Further longitudinal follow-up of this study sample is mandated to determine the prognostic potential of segmental wall thickness.

\section{Conclusion}

Our findings highlight the role of glycemic status as a potential risk factor and implicate prediabetes as unfavorably associated to LV wall thickness. Measurements of regional wall thickness provides a more precise picture than assessing overall myocardial mass. Delaying or halting progression from impaired fasting glucose to diabetes might prevent further thickening of the ventricular walls and subsequent cardiovascular complications.

\section{Additional file}

Additional file 1: Text S1. Description of laboratory measurements. Figure S1. Intra-and interobserver agreement. Text S2. Description of the underlying eligible cohort. Table S1. Characteristics of study subjects from the full eligible cohort used for the calculation of sampling weights. Table S2. Associations of glycemic status with wall thickness from weighted and unweighted linear regression models. Figure S2. Mean wall thickness according to prediabetic glycemic status. Table S3. Association of prediabetic glycemic status and mean wall thickness. (DOCX $57 \mathrm{~kb})$

\section{Abbreviations}

(i)IFG: (isolated) impaired fasting glucose; (i)IGT: (isolated) impaired glucose tolerance; AHA: American Heart Association; BMI: Body Mass Index; Cl: Confidence interval; CMR: Cardiac magnetic resonance; KORA: Cooperative Health Research in the Region of Augsburg; LV: Left ventricular; MRI: Magnetic resonance imaging; OGTT: Oral glucose tolerance test; SSFP: Steady-state free precession; WHO: World Health Organization

\section{Acknowledgements}

The authors wish to thank Mainsi Marowsky-Köppl and Andrea Wulff for expert data handling.

\section{Funding}

The KORA study was initiated and financed by the Helmholtz Zentrum München - German Research Center for Environmental Health, which is funded by the German Federal Ministry of Education and Research (BMBF) and by the State of Bavaria. Furthermore, KORA research was supported within the Munich Center of Health Sciences (MC-Health), LudwigMaximilians-Universität, as part of LMUinnovativ.

\section{Availability of data and materials}

The informed consent given by KORA study participants does not cover data posting in public databases. However, data are available upon request from KORA-gen (http://epi.helmholtz-muenchen.de/kora-gen/) by means of a project agreement. Requests should be sent to kora.passt@helmholtzmuenchen.de and are subject to approval by the KORA Board.

\section{Authors' contributions}

SR performed the statistical analyses, evaluated the results and drafted the paper. AS, $\mathrm{HH}$ and FB collected the CMR data and analyzed the images. $\mathrm{CH}, \mathrm{WR}, \mathrm{WK}, \mathrm{MH}, \mathrm{CM}, \mathrm{RL}, \mathrm{BL}, \mathrm{SA}$ and $\mathrm{HH}$ contributed substantially to data preparation and quality assurance. $\mathrm{FB}, \mathrm{AP}, \mathrm{HH}$ and $\mathrm{SA}$ participated in the conception and design of the study. WK, WR, CH, RL, BL, MH, CM, HH and AP revised the paper for important intellectual content. All authors have read and approved the final manuscript.

\section{Ethics approval and consent to participate}

The KORA FF4 study was approved by the ethics committee of the Bavarian Chamber of Physicians, Munich; the MRI substudy by the institutional review board of the Ludwig-Maximilians-University Munich. The investigations were carried out in accordance with the Declaration of Helsinki, including written informed consent of all participants.

\section{Consent for publication}

Not applicable.

\section{Competing interests}

The authors declare that they have no competing interests.

\section{Publisher's Note}

Springer Nature remains neutral with regard to jurisdictional claims in published maps and institutional affiliations.

\section{Author details}

${ }^{1}$ Institute of Epidemiology, Helmholtz Zentrum München, German Research Center for Environmental Health, Ingolstaedter Landstrasse 1, 85764 Neuherberg, Germany. ${ }^{2}$ Department of Radiology, Ludwig-Maximilians-University Hospital, Munich, Germany. ${ }^{3}$ Department of Internal Medicine II - Cardiology, University of Ulm Medical Center, UIm, Germany. ${ }^{4}$ Deutsches Herzzentrum München, Technische Universität München, Munich, Germany. ${ }^{5}$ German Centre for Cardiovascular Research (DZHK e.V.), Munich, Germany. ${ }^{6}$ German Center for Diabetes Research (DZD), München-Neuherberg, Germany. Institute for Biometrics and Epidemiology, German Diabetes Center, Duesseldorf, Germany. ${ }^{8}$ KORA Myocardial Infarction Registry, Central Hospital of Augsburg, Augsburg, Germany. ${ }^{9}$ Department of Diagnostic and Interventional Radiology, University of Tuebingen, Tuebingen, Germany.

Received: 29 January 2018 Accepted: 31 July 2018

Published online: 09 August 2018

\section{References}

1. Tsao CW, Gona PN, Salton CJ, Chuang ML, Levy D, Manning WJ, O'Donnell CJ. Left ventricular structure and risk of cardiovascular events: a Framingham heart study cardiac magnetic resonance study. J Am Heart Assoc. 2015;4(9):e002188.

2. Bluemke DA, Kronmal RA, Lima JAC, Liu K, Olson J, Burke GL, Folsom AR. The relationship of left ventricular mass and geometry to incident cardiovascular events. J Am Coll Cardiol. 2008;52(25):2148-55.

3. Burchfield JS, Xie M, Hill JA. Pathological ventricular remodeling: mechanisms: part 1 of 2. Circulation. 2013;128(4):388-400.

4. Edvardsen $T$, Rosen BD, Pan L, Jerosch-Herold M, Lai S, Hundley WG, Sinha S, Kronmal RA, Bluemke DA, Lima JA. Regional diastolic dysfunction in 
individuals with left ventricular hypertrophy measured by tagged magnetic resonance imaging--the multi-ethnic study of atherosclerosis (MESA). Am Heart J. 2006;151(1):109-14.

5. Kuznetsova T, Thijs L, Knez J, Herbots L, Zhang Z, Staessen JA. Prognostic Value of Left Ventricular Diastolic Dysfunction in a General Population. J Am Heart Assoc. 2014;3(3):e000789.

6. Devereux RB, Roman MJ. Left ventricular hypertrophy in hypertension: stimuli, patterns, and consequences. Hypertens Res. 1999;22(1):1-9.

7. De Simone G, Palmieri V, Bella JN, Celentano A, Hong Y, Oberman A, Kitzman DW, Hopkins PN, Arnett DK, Devereux RB. Association of left ventricular hypertrophy with metabolic risk factors: the HyperGEN study. J Hypertens. 2002;20(2):323-31.

8. Al-Daydamony MM, El-Tahlawi M. What is the effect of metabolic syndrome without hypertension on left ventricular hypertrophy? Echocardiography. 2016;33(9):1284-9.

9. Cuspidi C, Sala C, Negri F, Mancia G, Morganti A. Prevalence of leftventricular hypertrophy in hypertension: an updated review of echocardiographic studies. J Hum Hypertens. 2012;26(6):343-9.

10. Rutter MK, Parise H, Benjamin EJ, Levy D, Larson MG, Meigs JB, Nesto RW, Wilson PWF, Vasan RS. Impact of glucose intolerance and insulin resistance on cardiac structure and function: sex-related differences in the Framingham heart study. Circulation. 2003;107(3):448-54.

11. Palmieri V, Okin PM, de Simone G, Bella JN, Wachtell K, Gerdts E, Boman K, Nieminen MS, Dahlöf B, Devereux RB. Electrocardiographic characteristics and metabolic risk factors associated with inappropriately high left ventricular mass in patients with electrocardiographic left ventricular hypertrophy: the LIFE study. J Hypertens. 2007;25(5):1079-85.

12. Gerdts E, Okin PM, Omvik P, Wachtell K, Dahlöf B, Hildebrandt P, Nieminen MS, Devereux RB. Impact of diabetes on treatment-induced changes in left ventricular structure and function in hypertensive patients with left ventricular hypertrophy. The LIFE study. Nutr Metab Cardiovasc Dis. 2009;19(5):306-12.

13. Pareek M, Aharaz A, Nielsen ML, Gerke O, Leósdóttir M, Møller JE, Andersen $\mathrm{NH}$, Nilsson PM, Olsen MH. Untreated diabetes mellitus, but not impaired fasting glucose, is associated with increased left ventricular mass and concentric hypertrophy in an elderly, healthy, Swedish population. IJC Metabolic Endocrine. 2015;9:39-47.

14. Lorber R, Gidding SS, Daviglus ML, Colangelo LA, Liu K, Gardin JM. Influence of systolic blood pressure and body mass index on left ventricular structure in healthy African-American and white young adults: the CARDIA study. J Am Coll Cardiol. 2003;41(6):955-60.

15. Demmer RT, Allison MA, Cai J, Kaplan RC, Desai AA, Hurwitz BE, Newman JC, Shah SJ, Swett K, Talavera GA, et al. Association of Impaired Glucose Regulation and Insulin Resistance With Cardiac Structure and Function: Results From ECHO-SOL (Echocardiographic Study of Latinos). Circ Cardiovasc Imaging. 2016:9(10).

16. Cioffi G, Rossi A, Zoppini G, Targher G, de Simone G, Devereux RB, Vassanelli C, Bonora E. Inappropriate left ventricular mass independently predicts cardiovascular mortality in patients with type 2 diabetes. Int J Cardiol. 2013;168(5):4953-6.

17. Hoang K, Zhao Y, Gardin JM, Carnethon M, Mukamal K, Yanez D, Wong ND. LV mass as a predictor of CVD events in older adults with and without metabolic syndrome and diabetes. JACC Cardiovasc Imaging. 2015;8(9): 1007-15.

18. Eguchi K, Ishikawa J, Hoshide S, Ishikawa S, Pickering TG, Schwartz JE, Homma S, Shimada K, Kario K. Differential impact of left ventricular mass and relative wall thickness on cardiovascular prognosis in diabetic and nondiabetic hypertensive subjects. Am Heart J. 2007;154(1):79 e79-15.

19. Armstrong AC, Gidding S, Gjesdal O, Wu C, Bluemke DA, Lima JA. LVM assessed by echocardiography and cardiac magnetic resonance, cardiovascular outcomes, and medical practice. JACC Cardiovascular Imaging. 2012;5(8):837.

20. Bottini PB, Carr AA, Prisant LM, Flickinger FW, Allison JD, Gottdiener JS. Magnetic resonance imaging compared to echocardiography to assess left ventricular mass in the hypertensive patient. Am J Hypertens. 1995;8(3):221-8.

21. Bamberg F, Hetterich H, Rospleszcz S, Lorbeer R, Auweter SD, Schlett CL, Schafnitzel A, Bayerl C, Schindler A, Saam T, et al. Subclinical Disease Burden as Assessed by Whole-Body MRI in Subjects With Prediabetes, Subjects With Diabetes, and Normal Control Subjects From the General Population: The KORA-MRI Study. Diabetes 2017;66(1):158-69
22. Holle R, Happich M, Löwel H, Wichmann H. KORA--a research platform for population based health research. Gesundheitswesen. 2005;67:S19-25.

23. World Health Organization (WHO). Definition, diagnosis and classification of diabetes mellitus and its complications. In: Report of a WHO Consultation, Department of Noncommunicable Disease Surveillance. Geneva: World Health Organization; 1999.

24. Cerqueira MD, Weissman NJ, Dilsizian V, Jacobs AK, Kaul S, Laskey WK, Pennell DJ, Rumberger JA, Ryan T, Verani MS. Standardized myocardial segmentation and nomenclature for tomographic imaging of the heart a statement for healthcare professionals from the cardiac imaging committee of the council on clinical cardiology of the American Heart Association. Circulation. 2002;105(4):539-42.

25. Solon G, Haider SJ, Wooldridge JM. What are we weighting for? J Hum Resour. 2015;50(2):301-16.

26. Lieb W, Gona P, Larson MG, Aragam J, Zile MR, Cheng S, Benjamin EJ, Vasan RS. The natural history of left ventricular geometry in the community: clinical correlates and prognostic significance of change in LV geometric pattern. JACC CardiovasC Imaging. 2014;7(9):870-8.

27. Skali H, Shah A, Gupta DK, Cheng S, Claggett B, Liu J, Bello N, Aguilar D, Vardeny O, Matsushita K. Cardiac structure and function across the glycemic Spectrum in elderly men and women free of prevalent heart disease the atherosclerosis risk in the community study. Circ Heart Fail. 2015;8(3):448-54.

28. Velagaleti RS, Gona P, Chuang ML, Salton CJ, Fox CS, Blease SJ, Yeon SB, Manning WJ, O'Donnell CJ. Relations of insulin resistance and glycemic abnormalities to cardiovascular magnetic resonance measures of cardiac structure and function: the Framingham heart study. Circ Cardiovasc Imaging. 2010;3(3):257-63.

29. Heckbert SR, Post W, Pearson GD, Arnett DK, Gomes AS, Jerosch-Herold M, Hundley WG, Lima JA, Bluemke DA. Traditional cardiovascular risk factors in relation to left ventricular mass, volume, and systolic function by cardiac magnetic resonance imaging: the multiethnic study of atherosclerosis. J Am Coll Cardiol. 2006:48(11):2285-92.

30. Bertoni AG, Goff DC, D’Agostino RB, Liu K, Hundley WG, Lima JA, Polak JF, Saad MF, Szklo M, Tracy RP. Diabetic cardiomyopathy and subclinical cardiovascular disease the multi-ethnic study of atherosclerosis (MESA). Diabetes Care. 2006;29(3):588-94.

31. Henry RMA, Kamp O, Kostense PJ, Spijkerman AMW, Dekker JM, van Eijck R, Nijpels G, Heine RJ, Bouter LM, Stehouwer CDA. Left ventricular mass increases with deteriorating glucose tolerance, especially in women: independence of increased arterial stiffness or decreased flow-mediated dilation: the Hoorn study. Diabetes Care. 2004;27(2):522-9.

32. Kishi S, Gidding SS, Reis JP, Colangelo LA, Venkatesh BA, Armstrong AC, Isogawa A, Lewis CE, Wu C, Jacobs DR Jr, et al. Association of Insulin Resistance and Glycemic Metabolic Abnormalities with LV structure and function in middle age: the CARDIA study. JACC Cardiovasc Imaging. 2017;10(2):105-14.

33. Capaldo B, Di Bonito P, laccarino M, Roman MJ, Lee ET, Devereux RB, Riccardi G, Howard BV, de Simone G. Cardiovascular characteristics in subjects with increasing levels of abnormal glucose regulation: the strong heart study. Diabetes Care. 2013;36(4):992-7.

34. Bella JN, Devereux RB, Roman MJ, Palmieri V, Liu JE, Paranicas M, Welty TK, Lee ET, Fabsitz RR, Howard BV. Separate and joint effects of systemic hypertension and diabetes mellitus on left ventricular structure and function in American Indians (the strong heart study). Am J Cardiol. 2001;87(11):1260-5.

35. Palmieri V, Bella JN, Arnett DK, Liu JE, Oberman A, Schuck M-Y, Kitzman DW, Hopkins PN, Morgan D, Rao D. Effect of type 2 diabetes mellitus on left ventricular geometry and systolic function in hypertensive subjects. Circulation. 2001;103(1):102-7.

36. Li T, Chen S, Guo X, Yang J, Sun Y. Impact of hypertension with or without diabetes on left ventricular remodeling in rural Chinese population: a cross-sectional study. BMC Cardiovasc Disord. 2017;17(1):206.

37. Pierdomenico SD, Cuccurullo F. Risk reduction after regression of echocardiographic left ventricular hypertrophy in hypertension: a metaanalysis. Am J Hypertens. 2010;23(8):876-81.

38. Lonnebakken MT, Izzo R, Mancusi C, Gerdts E, Losi MA, Canciello G, Giugliano G, De Luca N, Trimarco B, de Simone G. Left Ventricular Hypertrophy Regression During Antihypertensive Treatment in an Outpatient Clinic (the Campania Salute Network). J Am Heart Assoc. 2017; 6(3):e004152. 
39. Asbun J, Villarreal FJ. The pathogenesis of myocardial fibrosis in the setting of diabetic cardiomyopathy. J Am Coll Cardiol. 2006;47(4):693-700.

40. Van Heerebeek L, Hamdani N, Handoko ML, Falcao-Pires I, Musters RJ, Kupreishvili K, ljsselmuiden AJ, Schalkwijk CG, Bronzwaer JG, Diamant M. Diastolic stiffness of the failing diabetic heart. Circulation. 2008;117(1):43-51.

41. Larghat AM, Swoboda PP, Biglands JD, Kearney MT, Greenwood JP, Plein S. The microvascular effects of insulin resistance and diabetes on cardiac structure, function, and perfusion: a cardiovascular magnetic resonance study. European Heart Journal-Cardiovascular Imaging. 2014;15(12):1368-76.

42. Levelt E, Mahmod M, Piechnik SK, Ariga R, Francis JM, Rodgers CT, Clarke WT, Sabharwal N, Schneider JE, Karamitsos TD, et al. Relationship between left ventricular structural and metabolic remodeling in type 2 diabetes. Diabetes. 2016;65(1):44-52.

43. Kawel N, Turkbey EB, Carr JJ, Eng J, Gomes AS, Hundley WG, Johnson C, Masri SC, Prince MR, van der Geest RJ, et al. Normal left ventricular myocardial thickness for middle-aged and older subjects with steady-state free precession cardiac magnetic resonance: the multi-ethnic study of atherosclerosis. Circulation: Cardiovascular Imaging. 2012;5(4):500-8.

44. Le Ven F, Bibeau K, De Larochelliere E, Tizon-Marcos H, DeneaultBissonnette S, Pibarot P, Deschepper CF, Larose E. Cardiac morphology and function reference values derived from a large subset of healthy young Caucasian adults by magnetic resonance imaging. Eur Heart J Cardiovasc Imaging. 2015;17(9):981-90

Ready to submit your research? Choose BMC and benefit from:

- fast, convenient online submission

- thorough peer review by experienced researchers in your field

- rapid publication on acceptance

- support for research data, including large and complex data types

- gold Open Access which fosters wider collaboration and increased citations

- maximum visibility for your research: over $100 \mathrm{M}$ website views per year

At $\mathrm{BMC}$, research is always in progress.

Learn more biomedcentral.com/submissions 